\title{
On the Observed Rate of Convergence of an Iterative Method Applied to a Model Elliptic Difference Equation
}

\author{
By R. A. Nicolaides
}

Abstract. A proof is given of the fact that the rate of convergence of a multiple grid type of algorithm is $O\left(h^{1 / 2}\right)$ in the case of a model elliptic difference equation.

1. This note provides a theoretical explanation of the $O\left(h^{1 / 2}\right)$ rate of convergence observed in the application of a multiple grid method to a model finite difference Poisson problem. The method in question was proposed in [2] and some numerical results were given from which the $O\left(h^{1 / 2}\right)$ figure was inferred. This method consists of applying a second degree acceleration technique to the iterates from a linear stationary iterative method of the first degree. Only the latter involves anything new-it is the multiple grid part of the algorithm-and is all that will be considered here. More specifically, once the spectral properties of the iterating matrix of the first degree process are established, the properties of the composite algorithm can be deduced by standard techniques. These were discussed in [2] and well-known books and papers [1] , [3] , [4] may be consulted for more details. Use of these techniques will be made in Section 4. In Sections 2 and 3 the necessary theoretical preliminaries are developed.

2. The problem considered in [2] was the solution of the partial difference equation

$$
L_{h} u_{i j}^{h}=f_{i j}^{h}, \quad i, j=1,2, \ldots, n,
$$

on the $(n+2) \times(n+2)$ square grid $\Omega_{h}$ (with grid boundary $\left.\partial \Omega_{h}\right)$ of side length $\pi$ and grid points of the form

$$
P_{i j}=(i h, j h), \quad i, j=0,1, \ldots, n+1 ; \quad(n+1) h=\pi .
$$

$u_{i j}^{h}$ is the sought for grid function evaluated at $P_{i j}$ and $u_{i j}^{h}$ vanishes on $\partial \Omega_{h} f_{i j}^{h}$ is a given grid function and the operator $L_{h}$ is the discrete Laplacian operator defined below.

For grid functions $v^{h}$ defined on $\bar{\Omega}_{h}$ the following operators are required:

$$
\begin{array}{ll}
\Delta_{1}^{h} v_{i j}^{h}=h^{-1}\left(v_{i+1 j}^{h}-v_{i j}^{h}\right), & 0 \leqslant i \leqslant n, 0 \leqslant j \leqslant n+1, \\
\nabla_{1}^{h} v_{i j}^{h}=h^{-1}\left(v_{i j}^{h}-v_{i-1 j}^{h}\right), & 1 \leqslant i \leqslant n+1,0 \leqslant j \leqslant n+1,
\end{array}
$$


with corresponding definitions for $\Delta_{2}^{h}$ and $\nabla_{2}^{h}$ the forward and backward difference operators in the $y$ direction. In this notation the formula for summation by parts becomes

$$
\sum_{i=0}^{n} v_{i j}^{h} \Delta_{1}^{h} w_{i j}^{h}=\left.h^{-1} v_{i j}^{h} w_{i j}^{h}\right|_{i=0} ^{n+1}-\sum_{i=1}^{n+1} w_{i j}^{h} \nabla_{1}^{h} v_{i j}^{h}
$$

and the discrete Laplacian $L_{h}$ introduced above is defined by

$$
L_{h} v_{i j}^{h}=-\Delta_{1}^{h} \nabla_{1}^{h} v_{i j}^{h}-\Delta_{2}^{h} \nabla_{2}^{h} v_{i j}^{h}
$$

A notational convention that will be used from now on is that undefined difference quotients such as $\nabla_{1}^{h} u_{0 j}^{h}$ are defined to be zero. With this understanding it follows from (2.3) using (2.2) that if $u_{i j}^{h}$ vanishes on $\partial \Omega_{h}$, then

$$
\sum_{i, j=0}^{n+1} u_{i j}^{h} L_{h} u_{i j}^{h}=\sum_{i, j=0}^{n+1}\left[\left(\nabla_{1}^{h} u_{i j}^{h}\right)^{2}+\left(\nabla_{2}^{h} u_{i j}^{h}\right)^{2}\right]
$$

which of course is an analogue of a result widely used in the continuous case. Another definition is needed; if $v^{h}$ and $w^{h}$ are grid functions defined on some subset $\bar{\Gamma}_{h}$ of $\bar{\Omega}_{h}$, then

$$
\left(v^{h}, w^{h}\right)_{\bar{\Gamma}_{h}}=h^{2} \sum_{i, j \in \bar{\Gamma}_{h}} v_{i j}^{h} w_{i j}^{h}
$$

and also

$$
\left|v^{h}\right|_{\bar{\Gamma}_{h}}^{2}=\left(v^{h}, v^{h}\right)_{\bar{\Gamma}_{h}}^{2}
$$

The summation indices on (2.5) are to be taken over the set of points in $\bar{\Gamma}_{h}$. (2.4) then can be written as

$$
\left(u^{h}, L_{h} u^{h}\right)_{\bar{\Omega}_{h}}=\left|\nabla_{1}^{h} u^{h}\right|_{\bar{\Omega}_{h}}^{2}+\left|\nabla_{2}^{h} u^{h}\right|_{\bar{\Omega}_{h}}^{2}=\left|\Delta_{1}^{h} u^{h}\right|_{\bar{\Omega}_{h}}^{2}+\left|\Delta_{2}^{h} u^{h}\right|_{\bar{\Omega}_{h}}^{2}
$$

The notational convention introduced above is in action on both of these equations.

The lemma which follows is a discrete analogue of Poincarés inequality, essential to later arguments. The proof is little more than a discrete copy of a proof well known in the continuous case but will be given for completeness and because it is short.

LEMma 2.1 (PoINCARE). Let $\bar{\Gamma}_{h}$ be any $(n+2) \times(n+2)$ square grid with spacing $h=A /(n+1)$, and let $v^{h}$ be any grid function defined on $\bar{\Gamma}_{h}$. Then

$$
\left|v^{h}\right|_{\bar{\Gamma}_{h}}^{2} \leqslant A^{2}\left(\frac{n+2}{n+1}\right)\left[\left|\Delta_{1}^{h} v^{h}\right|_{\bar{\Gamma}_{h}}^{2}+\left|\Delta_{2}^{h} v^{h}\right|_{\bar{\Gamma}_{h}}^{2}\right]+(n+2)^{-2}\left[\sum_{i, j \in \bar{\Gamma}_{h}} v_{i j}^{h}\right]^{2} .
$$

Proof. Note. $h$ is used as a symbol here to avoid proliferation of notations. It is not related to the $h$ used earlier. 
Name the grid points $P_{i j}, i, j=0,1, \ldots, n+1$, in the usual way. It is clear that

$$
v_{i j}^{h}-v_{k j}^{h}=\operatorname{sgn}(i-k) h \sum_{\alpha=\min (i, k)}^{\max (i, k)-1} \Delta_{1}^{h} v_{\alpha j}^{h}
$$

and, consequently, that

$$
v_{i j}^{h}-v_{k l}^{h}=\operatorname{sgn}(i-k) h \sum_{\alpha=\min (i, k)}^{\max (i, k)-1} \Delta_{1}^{h} v_{\alpha j}^{h}+\operatorname{sgn}(j-l) h \sum_{\beta=\min (j, l)}^{\max (j, l)-1} \Delta_{2}^{h} v_{k \beta}^{h} .
$$

Squaring both sides, using Cauchy's inequality twice and increasing the summation indices yields

$$
\left(v_{i j}^{h}\right)^{2}+\left(v_{k l}^{h}\right)^{2}-2\left(v_{i j}^{h} v_{k l}^{h}\right) \leqslant 2 h^{2}(n+1)\left[\sum_{\alpha=0}^{n}\left(\Delta_{1}^{h} v_{\alpha j}^{h}\right)^{2}+\sum_{\beta=0}^{n}\left(\Delta_{2}^{h} v_{k \beta}^{h}\right)^{2}\right]
$$

Summing for $i, j=0,1, \ldots, n+1$, then for $k, l=0,1, \ldots, n+1$, gives

$$
\begin{aligned}
& (n+2)^{2}\left|v^{h}\right|_{\bar{\Gamma}_{h}}^{2}+(n+2)^{2}\left|v^{h}\right|_{\bar{\Gamma}_{h}}^{2}-2\left(\sum_{i, j \in \bar{\Gamma}_{h}} v_{i j}^{h}\right)^{2} \\
& \leqslant 2 h^{2}(n+1)(n+2)^{3}\left[\left|\Delta_{1}^{h} v^{h}\right|_{\bar{\Gamma}_{h}}^{2}+\left|\Delta_{2}^{h} v^{h}\right|_{\bar{\Gamma}_{h}}^{2}\right]
\end{aligned}
$$

which is equivalent to the stated result.

In the next section some facts about the iterative method under discussion are collected together.

3. To introduce the algorithm suggested in [2] , first of all, the difference equation (2.1) must be put into matrix form. To do this, an arbitrary fixed order is assigned to the grid points of $\bar{\Omega}_{h}$ and the equation (2.1) written for the points of $\Omega_{h}$ in the order of their listing. This is the usual procedure. The result of it is a system of linear equations, $N$ of them, which will be denoted by $A_{h} u^{h}=f^{h}$, where $A_{h}$ is an $N \times N$ positive definite matrix with $4 / h^{2}$ in each diagonal position, and the meaning of the vectors $u^{h}$ and $f^{h}$ is the obvious one.

No confusion will arise with the earlier use of these symbols. Secondly, the internal grid points are partitioned into subsets as follows; take $N=n^{2}$ for some positive integer $n$ so that there are $n^{4}$ points in $\Omega_{h}$, and divide them into $n^{2}$ sets of $n^{2}$ points by drawing $n^{2}-1$ lines equally spaced and parallel to the $x$ axis and again for the $y$ axis. List the subsets in some order and denote them by $\Omega_{h}^{(i)}, i=1,2, \ldots$, $n^{2}$. For any vector $v^{h}$ defined at the points of $\Omega_{h}$, a "contracted" vector $\bar{v}^{h}$ is defined by:

$$
\left(\bar{v}^{h}\right)_{i}=\frac{1}{n} \sum_{k \in \Omega_{h}^{(i)}}\left(v^{h}\right)_{k}, \quad i=1,2, \ldots, n^{2}
$$


This transformation may be represented by a matrix $\left(E_{h}\right)^{T}$, where $E_{h}$ has dimensions $n^{4} \times n^{2}$ and the $i$ th column of $E_{h}$, associated with $\Omega_{h}^{(i)}$, has $1 / n$ in those positions with numbers corresponding to grid points in $\Omega_{h}^{(i)}$ and zeros in the other positions. The matrix $E_{h}^{T} E_{h}$ will then be the unit matrix of order $n^{2} \times n^{2}$. With these notations, the matrix

$$
\left(I-E_{h}\left(E_{h}^{T} A_{h} E_{h}\right)^{-1} E_{h}^{T} A_{h}\right)\left(I-\frac{h^{2}}{4} A_{h}\right)
$$

was found in [2] to be the iterating matrix of the first degree method referred to in Section 1. The relevant spectral properties of (3.2) are deduced below.

Let

$$
\Pi_{h}=\left(I-E_{h}\left(E_{h}^{T} A_{h} E_{h}\right)^{-1} E_{h}^{T} A_{h}\right), \quad B_{h}=\left(I-\frac{h^{2}}{4} A_{h}\right)
$$

Lemma 3.1. The eigenvalues of $\Pi_{h} B_{h}$ are real.

Proof. Let $S_{h}^{2}=A_{h}$, with $S_{h}$ positive definite. Then $S_{h} \Pi_{h} S_{h}^{-1}$ is similar to $\Pi_{h}$ and is an orthogonal projection matrix. Also $S_{h} B_{h}=B_{h} S_{h}$ and so $\Pi_{h} B_{h}$ is similar to

$$
S_{h} \Pi_{h} S_{h}^{-1} B_{h}=\left(S_{h} \Pi_{h} S_{h}^{-1}\right)^{2} B_{h} .
$$

The latter has the same eigenvalues as the symmetric matrix

$$
\left(S_{h} \Pi_{h} S_{h}^{-1}\right) B_{h}\left(S_{h} \Pi_{h} S_{h}^{-1}\right)
$$

and the lemma follows.

Let $E_{h}$ denote $\operatorname{span}\left(E_{h}\right)$ and let $P_{h}=E_{h}\left(E_{h}^{T} E_{h}\right)^{-1} E_{h}^{T}$ denote the orthogonal projector onto $E_{h}$. Put also $Q_{h}=I-P_{h}$.

LEMMA 3.2. Let $w^{h} \in E_{h}^{1}$ Then the corresponding grid function $w_{i j}^{h}$ satisfies

$$
\sum_{i, j \in \Omega_{h}^{(k)}} \mathrm{w}_{i j}=0, \quad k=1,2, \ldots, n^{2}
$$

Proof. $w^{h}$ necessarily satisfies

$$
w^{h}=\left(I-E_{h}\left(E_{h}^{T} E_{h}\right)^{-1} E_{h}^{T}\right) s^{h} \equiv\left(I-E_{h} E_{h}^{T}\right) s^{h}
$$

for some vector $s^{h}$. The vector on the right consists of a vector $s^{h}$ minus a vector which is constant in each $\Omega_{h}^{(k)}$ and equal to the average value of $s^{h}$ over $\Omega_{h}^{(k)}$. So the average of $w^{h}$ is zero over each $\Omega_{h}^{(i)}$ and this is equivalent to (3.4).

The eigenvalues of $\Pi_{h} B_{h}, \gamma$, satisfy for some real vectors $\xi^{h}$,

$$
\Pi_{h} B_{h} \xi^{h}=\gamma \xi^{h}
$$

Multiplying on the left of (3.5) by $Q_{h}$ and using $Q_{h} E_{h}=0$ gives

$$
Q_{h} B_{h} \xi^{h}=\gamma Q_{h} \xi^{h}
$$


and multiplying on the left of (3.5) by $P_{h} A_{h}$ gives after a little simplification when $\gamma \neq 0$

$$
Q_{h} A_{h} \xi^{h}=A_{h} \xi^{h}
$$

Put $\delta=1-\gamma, \delta \neq 1$. Substituting from (3.3) for $B_{h}$ in (3.6), it follows that

$$
\frac{h^{2}}{4} Q_{h} A_{h} \zeta^{h}=\delta Q_{h} \zeta^{h}=\frac{h^{2}}{4} A_{h} \xi^{h}
$$

by (3.7).

Multiplying on the left by $A_{h}^{-1}$ and taking the (vector) inner product of both sides with $Q_{h} \zeta^{h}$, there results the equation for $\delta$,

$$
=\frac{h^{2}}{4} \frac{\left(Q_{h} \zeta^{h}, Q_{h} \zeta^{h}\right)}{\left(Q_{h} \zeta^{h}, A_{h}^{-1} Q_{h} \zeta^{h}\right)}
$$

where the property $Q_{h}^{T} Q_{h} \equiv Q_{h}^{2}=Q_{h}$ has been used. (3.8) will be used to bound $\gamma$ above and below. This will be done in Section 4 .

4. If $u_{i j}^{h}$ and $v_{i j}^{h}$ are grid functions vanishing on $\partial \Omega_{h}$, the following relations may be (trivially) verified

$$
\left(u^{h}, v^{h}\right)_{\bar{\Omega}_{h}}=h^{2}\left(u^{h}, v^{h}\right), \quad\left(u^{h}, L_{h} v^{h}\right)_{\bar{\Omega}_{h}}=h^{2}\left(u^{h}, A_{h} v^{h}\right)
$$

where the quantities in parentheses on the right are vector inner products. To obtain a lower bound for $\gamma$ the simple inequality

$$
\left(u^{h}, u^{h}\right)^{2} \leqslant\left(u^{h}, A_{h} u^{h}\right)\left(u^{h}, A_{h}^{-1} u^{h}\right)
$$

actually valid for any positive definite matrix, may be used. Thus,

$$
1-\gamma \leqslant \frac{h^{2}}{4} \frac{\left(u^{h}, A_{h} u^{h}\right)}{\left(u^{h}, u^{h}\right)}<2,
$$

the last coming, e.g. from Gershgorin's theorem (strengthened as in [3]). So then

$$
\gamma>-1 \text {. }
$$

By using other arguments, unnecessary here, it may be shown that $\gamma>-1+K h^{2}$.

This is well known. To obtain the upper bound is less simple.

To begin with, define $w^{h}$ by $A_{h} w^{h}=Q_{h} \xi^{h}$, where $Q_{h} \xi^{h}$ appears in (3.8).

Then (3.8) becomes

$$
\delta=\frac{h^{2}}{4} \frac{\left(A_{h} w^{h}, A_{h} w^{h}\right)}{\left(w^{h}, A_{h} w^{h}\right)} \geqslant \min _{A_{h} w^{h} \in E_{h}^{\frac{1}{h}}} \frac{h^{2}}{4} \frac{\left(A_{h} w^{h}, A_{h} w^{h}\right)}{\left(w^{h}, A_{h} w^{h}\right)}
$$

This minimum value is estimated (actually sharply to within a multiplicative constant) in the following theorem.

THEOREM 4.1. Let $s^{h}$ be any vector such that $A_{h} s^{h} \in E_{h}^{1}$ and let $s_{i j}^{h}$ be the 
corresponding grid function vanishing on $\partial \Omega_{h}$. Then

$$
\left(L_{h} s^{h}, L_{h} s^{h}\right)_{\bar{\Omega}_{h}} \geqslant(\Pi h)^{-1}\left(s^{h}, L_{h} s^{h}\right)_{\bar{\Omega}_{h}} .
$$

Proof. Let $A_{h} s^{h}=w^{h} \in E_{h}^{1}$, and let $w_{i j}^{h}$ be the corresponding grid function vanishing on $\Omega_{h}$. Then $\left(s^{h}, A_{h} s^{h}\right)=\left(s^{h}, w^{h}\right)$. By (2.6), (4.1) and the last equation,

$$
\begin{aligned}
\left(s^{h}, L_{h} s^{h}\right)_{\bar{\Omega}_{h}} & =\left|\Delta_{1}^{h} s^{h}\right|_{\bar{\Omega}_{h}}^{2}+\left|\Delta_{2}^{h} s^{h}\right|_{\bar{\Omega}_{h}}^{2}\left|\Delta_{1}^{h} s^{h}\right|_{\bar{\Omega}_{h}}^{2}+\left|\Delta_{2}^{h} s^{h}\right|_{\bar{\Omega}_{h}}^{2} \\
& =\left(s^{h}, w^{h}\right)_{\bar{\Omega}_{h}}=h^{2} \sum_{k} \sum_{\bar{\Omega}_{h}^{(k)}} w_{i j}^{h} s_{i j}^{h} .
\end{aligned}
$$

Let $\bar{s}_{k}^{h}$ denote the mean of $s_{i j}^{h}$ over $\bar{\Omega}_{h}^{(k)}$, so that

$$
\overline{s_{k}^{h}}=\frac{1}{\left|\bar{\Omega}_{h}^{(k)}\right|} \sum_{i, j \in \bar{\Omega}_{h}^{(k)}} s_{i j}^{h}
$$

Then since $w^{h} \in E_{h}^{1}$ and by Lemma 3.2, it follows that

$$
\left|\Delta_{1}^{h} s^{h}\right|_{\bar{\Omega}_{h}}^{2}+\left|\Delta_{2}^{h} s^{h}\right|_{\bar{\Omega}_{h}}^{2}=h^{2} \sum_{k} \sum_{\bar{\Omega}_{h}^{(k)}} w_{i j}^{h}\left(s_{i j}^{h}-\bar{s}_{k}^{h}\right) .
$$

By Lemma 2.1 , with $A^{2}=\left[(n-1) \pi /\left(n^{2}+1\right)\right]^{2}$, and making use of the vanishing over $\bar{\Omega}_{h}^{(k)}$ of $\Sigma\left(s_{i j}^{h}-\bar{s}_{k}^{h}\right)$, one has

$$
\left|s^{h}-\bar{s}\right|_{\bar{\Omega}_{h}^{(k)}}^{2} \leqslant\left(\frac{n}{n-1}\right) A^{2}\left(\left|\Delta_{1}^{h}\left(s^{h}-\bar{s}^{h}\right)\right|_{\bar{\Omega}_{h}^{(k)}}^{2}+\left|\Delta_{2}^{h}\left(s^{h}-\bar{s}^{h}\right)\right|_{\bar{\Omega}_{h}^{(k)}}^{2}\right)
$$

and furthermore,

$$
\left|\Delta_{i}^{h}\left(s^{h}-\bar{s}^{h}\right)\right|_{\bar{\Omega}_{h}^{(k)}}^{2}=\left|\Delta_{i}^{h} s^{h}\right|_{\bar{\Omega}_{h}^{(k)}}^{2}, \quad i=1,2 .
$$

Applying Cauchy's inequality to (4.3) and using (4.4) and (4.5),

$$
\begin{aligned}
\left|\Delta_{1}^{h} s^{h}\right|_{\bar{\Omega}_{h}}^{2}+\left|\Delta_{2}^{h} s^{h}\right|_{\bar{\Omega}_{h}}^{2} \leqslant h^{2} \sum_{k}\left(\sum_{\bar{\Omega}_{h}^{(k)}}\left(w_{i j}^{h}\right)^{2}\right)^{1 / 2}\left(\sum_{\bar{\Omega}_{h}^{(k)}}\left(s_{i j}^{h}-\bar{s}_{k}^{h}\right)^{2}\right)^{1 / 2} \\
\quad=\sum_{k}\left|w^{h}\right|_{\bar{\Omega}_{h}^{(k)}}\left|s^{h}-\bar{s}^{h}\right|_{\bar{\Omega}_{h}^{(k)}} \\
\\
\leqslant\left(\frac{n+1}{n}\right)^{1 / 2} A \sum_{k}\left|w^{h}\right|_{\bar{\Omega}_{h}^{(k)}}\left[\left|\Delta_{1}^{h} s^{h}\right|_{\bar{\Omega}_{h}^{(k)}}^{2}+\left|\Delta_{2}^{h} s^{h}\right|_{\bar{\Omega}_{h}^{(k)}}^{2}\right]^{1 / 2} \\
\leqslant\left(\frac{n+1}{n}\right)^{1 / 2} A\left|w^{h}\right|_{\bar{\Omega}_{h}}\left[\left|\Delta_{1}^{h} s^{h}\right|_{\bar{\Omega}_{h}}^{2}+\left|\Delta_{2}^{h} s^{h}\right|_{\bar{\Omega}_{h}}^{2}\right]^{1 / 2}
\end{aligned}
$$

by Cauchy's inequality again, and using the fact that 


$$
\left(\sum_{k}\left|\Delta_{1}^{h} s^{h}\right|_{\bar{\Omega}_{h}^{(k)}}^{2}+\left|\Delta_{2}^{h} s^{h}\right|_{\bar{\Omega}_{h}^{(k)}}^{2}\right)^{1 / 2} \leqslant\left(\left|\Delta_{1}^{h} s^{h}\right|_{\bar{\Omega}_{h}}^{2}+\left|\Delta_{2}^{h} s^{h}\right|_{\bar{\Omega}_{h}}^{2}\right)^{1 / 2},
$$

this reduces to

$$
\left|\Delta_{1}^{h} s^{h}\right|_{\bar{\Omega}_{h}}^{2}+\left|\Delta_{2}^{h} s^{h}\right|_{\bar{\Omega}_{h}}^{2} \leqslant\left(\frac{n}{n-1}\right) A^{2}\left|w^{h}\right|_{\bar{\Omega}_{h}}^{2} \leqslant \pi h\left|w^{h}\right|_{\bar{\Omega}_{h}}^{2} ;
$$

and since $\left(w^{h}, w^{h}\right)_{\bar{\Omega}_{h}}=\left(L_{h} s^{h}, L_{h} s^{h}\right)_{\bar{\Omega}_{h}}^{2}$, the result is proved.

It now follows, making use of the expression for $\delta$ given before the statement of Theorem 4.1 , that

$$
\delta \geqslant \frac{h^{2}}{4} \cdot \frac{1}{\pi h}=\frac{h}{4 \pi}
$$

and finally that

$$
\gamma \leqslant 1-\frac{h}{4 \pi}
$$

Thus, all the eigenvalues of $\Pi_{h} B_{h}$ are contained within the interval $(-1,1-h / 4 \pi)$. It will now be shown that the composite iteration consisting of applying the second degree method to the iterates from $\Pi_{h} B_{h}$ converges at the rate $O\left(h^{1 / 2}\right)$. It is known that to do this, the quantity

$$
\bar{\mu}=\frac{[1-(-1)]-[(1-(1-h / 4 \pi))]}{[1-(-1)]+[(1-(1-h / 4 \pi))]}
$$

and its square are formed. The effective rate of convergence is then given by

$$
\ln \left(\frac{1-\sqrt{ }\left(1-\bar{\mu}^{2}\right)}{1+\sqrt{ }\left(1-\bar{\mu}^{2}\right)}\right)^{1 / 2}
$$

Expanding in series and neglecting all but the smallest powers of $h$ gives for this latter the required $O\left(h^{1 / 2}\right)$ figure. The quantity called in [2] the effective spectral radius is similarly computed and turns out to be $1-h^{1 / 2} / \sqrt{2 \pi}$. The empirically observed value was $1-2 h^{1 / 2} / \sqrt{\pi}$.

Finally, it seems reasonable that the results found above could be adapted to generalizing the rate of convergence result to other second order elliptic problems.

Department of Mathematics

Imperial College of Science and Technology

180 Queen's Gate

London SW7, England

1. G. H. GOLUB \& R. S. VARGA, " Chebychev semi-iterative methods, SOR methods and second order Richardson methods," Numer. Math., v. 3, 1961, pp. 147-168.

2. R. A. NICOLAIDES, "On multiple grid and related techniques for the solution of discrete elliptic systems," J. Computational Phys., v. 19, 1975, pp. 418-431.

3. R. S. VARGA, Matrix Iterative Analysis, Prentice-Hall, Englewood Cliffs, N. J., 1962.

4. D. M. YOUNG, Iterative Solution of Large Linear Systems, Academic Press, New York and London, 1971. 